\title{
Instantaneous sentinel for the identification of the pollution term in Navier-Stokes system *
}

\author{
Laib Teldja \\ University of Larbi Ben M'hidi Oum El Bouaghi, Algeria \\ Rezzoug Imad \\ University of Larbi Ben M'hidi Oum El Bouaghi., Algeria and \\ Oussaeif Taki-Eddine \\ LUniversity of Larbi Ben M'hidi Oum El Bouaghi., Algeria \\ Received : June 2020. Accepted : June 2021
}

\begin{abstract}
The aim of this paper is about presenting some results of the Sentinel Theory in Connection with Control Theory of Distributed Systems. There is of course a large variety of models where the results to follow could be applied. What we have particularly in mind is the classical set of Navier-Stokes equations. We shall denote here by the velocity field and the pressure, which is a quite unusual notation in the "Turbulence" circle. The reason is simply that in all what is following that we think of as the state of our system, this state depends on control functions, these control functions are being either "artificial" or "natural". Also in this work we are(trying) working to get instantaneous information at fixed instant "T" on pollution term in Navier-Stokes system in which the initial condition is incomplete. The best method which can solve this problem is the sentinel one; it allows the estimation of the pollution term at which we look for information independently of the missing term that we do not want to identify. So, we prove the existence of such instantaneous sentinel by solving a problem of controllability with a constraint on the control.
\end{abstract}

2010 Mathematics Subject Classification: Primary 93B05; Secondary 93C20, 92D40.

Key words and phrases: Control Theory, Navier Stokes system with incomplete data, Turbulence Theory, Instantaneous Sentinel.

*This work was supported by Prof Ayadi Abdelhamid 


\section{Introduction}

The aim of this paper is to present some findings and remarks to some extent on turbulence theory in connection with control theory of distributed systems as well as how to apply the sentinel method to assess the amount of pollution term.

The first remarks, address the question of using control theory to derive possibly optimal, numerical algorithms. One has of course to make precise what is meant by optimal, i.e. to define a "cost function" in the terminology of control theory. In general terms, one wants to find algorithms which give the information we are looking for and which "suppress" the difficulties introduced by turbulence or chaos.

The second remarks, address the question of controlling turbulence. This situation arises if we can act on the system through control functions. Several approaches are, at least theoretically, conceivable. They also depend on the "cost function". We consider two families of "cost functions". The first one to be considered expresses the "mixing" of scales which is one of the properties of turbulence. The second family of "cost functions" is connected with exact controllability.

There is of course a large variety of models where the remarks to follow would apply.

What we have particularly in mind is the classical set of Navier-Stokes equations.

We shall denote here by $y$ the velocity field and by $\Pi$ the pressure, a quite unusual notation in the turbulence circle. From now on $y_{0}$ and $f$ are sufficiently small (in the neighborhood of zero), so the problem has a unique solution (see 5[3],[11], [4]])

The reason is simply that in all what follows we think of $y$ as the state of our system, this state depending on control functions, these control functions being either artificial or natural.

We shall write therefore for the state equation :

(1.1) $\left\{\begin{aligned} \frac{\partial y}{\partial t}+(y \nabla) y-\Delta y+f(y) & =-\nabla \Pi & & \text { in } Q=\Omega \times(0, T), \\ \text { divy } & =0 & & \text { in } Q,\end{aligned}\right.$

we assume here that $f: R^{n} \longrightarrow R^{n}, n=2,3$ is of class $C^{1 \text {. }}$

Subject to initial conditions and to boundary conditions.

In (1.1) $\Omega$ is a bounded open set of $R^{n}, n=2,3$.

The control functions, when there are of the "natural" type, will be either in the "source terms", in the right hand side of (1.1), or on the 
boundary ; more precisely

$$
\left\{\begin{aligned}
\frac{\partial y}{\partial t}+(y \nabla) y-\Delta y+f(y) & =-\nabla \Pi+\chi_{O} & & \text { in } Q=\Omega \times(0, T), \\
\operatorname{divy} & =0 & & \text { in } \quad Q \\
y & =0 & & \text { on } \Sigma=\partial \Omega \times(0, T),
\end{aligned}\right.
$$

where

$$
\begin{aligned}
O & =\text { open set contained in } \Omega, \\
\chi_{O} & =\text { characteristic function of } O, \\
u=u(x, t) & =\text { control function. }
\end{aligned}
$$

Equations (1.2) express the fact that we can act on the system in the region $O$ (not necessarily connected) and which can be "very small".

According to Lesieur [18] it means that the system has two properties

$$
\text { "the" state } y \text { sudden "unpredictable" }
$$

the system satisfies the "increased mixing property"

(the flow is able to mix transported quantities much more rapidly than if only molecular diffusion processes were involved).

In order to make (1.3) more precise, we introduce initial conditions in a particular form. We shall assume that

$$
y(x, 0)=y_{0}(x)+\tau \widehat{y}_{0}(x) \text { in } \Omega,
$$

the function $y_{0}$ is known with $y_{0} \in H H=$ Hilbert space. But, the term : $\tau \widehat{y}_{0}$ (so-called missing term) is unknown, with norm 1 , and where $\tau \in R$ is small.

We shall assume that "the" state $y$ is "very sensitive" to $\tau$. So the equations (1.2) and (1.5) uniquely define the solution

$$
y=y\left(x, t ; \tau \widehat{y}_{0}, u\right) .
$$

The "contradiction" is apparent since the strategy that we shall (try to) follow is to derive equations based on (1.6) and on control theory. But these equations are meaningful independently of (1.6) and we will (try to) study directly these equations.

It remains to make precise hypothesis (1.3), (1.4) using "the" state as in (1.6). 


\section{Approximation Methods}

The initial condition is given by

$$
y(x, 0)=y_{0}(x) \text { in } \Omega .
$$

One way (of course not the only one !) to look at this is to rewrite (1.1) under the form

$$
\frac{\partial y}{\partial t}+(y \nabla) y-\Delta y+w=0
$$

with the constraint

$$
w=f(y) .
$$

The next step is to relax (2.3). Let us consider a functional $\phi(y)$ where we think of $y$, for the time being, as "the" solution of (1.2), (2.1) and that we would like to keep "small".

Example 1. If we do not want to consider turbulence on $O \subset \Omega$ and during the time interval $\left(t_{0}, T\right)$, we could take

$$
\phi(y)=\iint_{O \times\left(t_{0}, T\right)}(\operatorname{curl} y)^{2} d x d t
$$

for example curl contours and curl in the surface stress.

The problem of optimal control we introduce is now the following.

Let $y(w)$ be the solution of $(2.2),(2.1),(1.2)$. We introduce

$$
J_{k}(w)=\phi(y(w))+k \int_{\Omega \times(0, T)}(w-f(y))^{2} d x d t,
$$

where $k>0$ plays the role of a penalty term.

We consider then the problem

$$
\inf _{w \in L^{2}(O \times(0, T))} J_{k}(w) .
$$

Remark 1. One can write the optimality system corresponding to problem (2.6). One can add constraints of other types on $w$. And very many variants are of course possible. One can in particular decompose the solution $y$ in two parts, say $y=z+\zeta$ and apply the above ideas to one part, say $\zeta$. 


\section{Control of Turbulence}

Let us consider now (1.2), (1.5) and let us assume for the time being that (1.6) holds true. We assume that we are in the situation (1.3), (1.4).

Let us consider a functional $\phi_{1}(y)$ which is, in principle, "unpredictable" if there are small variations in the initial condition (1.5).

We shall say that the control $u$ is insensitive with respect to the functional $\phi_{1}$ if

$$
\left.\frac{d}{d \tau} \phi_{1}(y(\tau, u))\right|_{\tau=0}=0, \quad \forall \widehat{y}_{0}
$$

In (3.1), we have written $y(\tau, u)$ for $y\left(x, t ; \tau \widehat{y}_{0}, u\right)$ and we assume that the $\tau$ derivative does exist near the origin.

If $f$ and $\phi_{1}$ are smooth, then (3.1) is equivalent to

$$
\left(\phi_{1}^{\prime}(y(u)), y_{\tau}\right)=0, \quad \forall \widehat{y}_{0} .
$$

Where

$$
\begin{gathered}
\phi_{1}^{\prime}=\quad \text { Frechet's derivative of } \phi_{1}, \\
y(u)=\quad \text { solution of }(1.2) \text { and }(1.5) \text { where } \tau=0, \\
y_{\tau}=\left.\frac{d}{d \tau} y\left(x, t ; \tau \widehat{y}_{0}, u\right)\right|_{\tau=0} .
\end{gathered}
$$

If $f$ is smooth, $y_{\tau}$ is given by

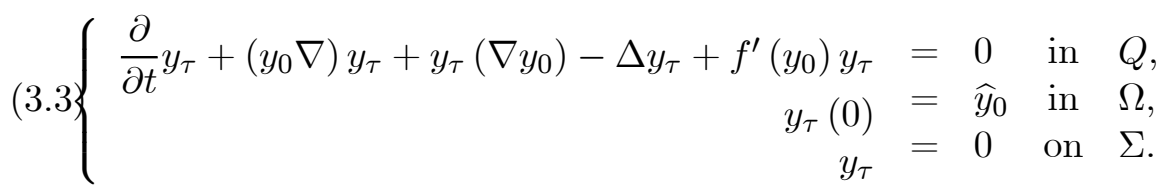

Where $f^{\prime}\left(y_{0}\right)$ denotes the derivative of $f$ at point $y_{0}$ and where $y_{0}$ is the solution of the problem

$\frac{\partial y_{0}}{\partial t}+\left(y_{0} \nabla\right) y_{0}-\Delta y_{0}+f\left(y_{0}\right)=-\nabla \Pi$ in $Q, y_{0}=0$ on $\Sigma, y_{0}(0)=y_{0}$ in $\Omega$.

We restrict the choice of $u$ to those controls - provided they exist - which satisfy (3.2), the insensitive controls.

We consider next a second functional $\phi_{2}(y)$ which, in principle, is related to the "increased mixing property" (1.4).

For instance, in the case of Navier-Stokes equations, we could consider

$$
\phi_{2}(y)=\phi(y) \quad \text { as given by }(2.4) \text {. }
$$


Then the problem of controlling turbulence can be expressed as

$$
\inf _{u \in L^{2}(O \times(0, T))} \phi_{2}(y(u)),
$$

where

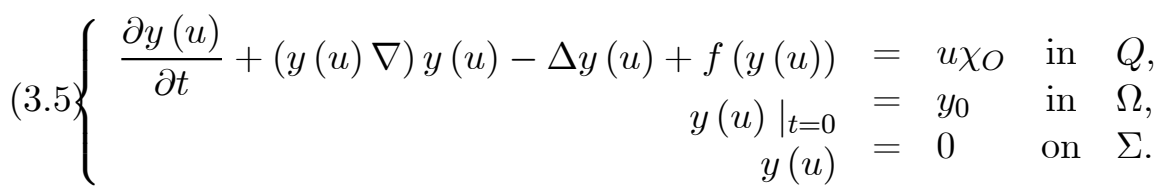

And where $u$ is subject to (3.2) (insensitivity).

We proceed by transforming (3.2). To this effect, we introduce as it is classical in sensitivity studies, the adjoint state $q$ defined by

(3.6 $\left\{\begin{aligned}-\frac{\partial q}{\partial t}+\left(y_{0} \nabla\right) q+q\left(\nabla y_{0}\right)-\Delta q+f^{\prime}\left(y_{0}\right)^{*} q & =\phi_{1}^{\prime}(y) & \text { in } Q, \\ q(x, T) & =0 & \text { in } \Omega, \\ q & =0 & \text { on } \Sigma .\end{aligned}\right.$

Then (3.2) becomes, using (3.3) and integration by parts

$$
\int_{\Omega} q(x, 0) d x=0, \quad \forall \widehat{y}_{0},
$$

such that $q(x, 0)=\widehat{y}_{0}(x)$,i.e.

$$
q(x, 0)=0 \text { in } \Omega .
$$

The insensitive controls with respect to $\phi_{1}$ are those which are such that (3.5), (3.6), (3.8) hold true.

Remark 2. The existence of such insensitive controls seems to be in general a non trivial problem (see [14]).

The problem can now be formulated, find

$$
\inf _{u \in L^{2}(\Omega \times(0, T))} \phi_{2}(y(u)), \quad u \quad \text { subject to }(3.5),(3.6),(3.8) .
$$

A quite realistic way to avoid the difficulty indicated in Remark 2 (but of course the question raised in this Remark remains of interest, at least we think !) is to relax (3.8) and to consider

$$
\inf _{u \in L^{2}(\Omega \times(0, T))}\left[\phi_{2}(y(u))+k \int_{\Omega} q(x, 0)^{2} d x\right], \quad k>0 .
$$

In this formulation one can add other constraints on $u$. 


\section{Exact controllability}

Let us now consider the equations

$$
\frac{\partial y}{\partial t}+(y \nabla) y-\Delta y+f(y)=u \chi_{O},
$$

subject to

$$
y=0 \quad \text { on } \quad \Gamma \times(0, T),
$$

and where

$$
y(0)=y_{0} \quad \text { is given } .
$$

We formulate the problem of exact controllability in a way which makes sense even if existence and uniqueness of a solution of (4.1), (4.2) and (4.3) is not known.

The first formulation is as follows: let $T$ be given and let $z_{0}$ be given in a suitable function space. We say that the system is exactly controllable at time $T$ if for every couple $y_{0}, z_{0}$, there exists a couple $(u, y)$ satisfying (4.1), (4.2) and (4.3) such that

$$
y(T)=z_{0} \quad, \quad(y(T)=y(x, T)) .
$$

Moreover, if there is exact controllability, one wants to find $u$ such that (4.4) holds true and which minimizes (for instance)

$$
\iint_{O \times(0, T)} u^{2} d x d t
$$

After J.L. Lions, this formulation may be too restrictive and it can be replaced by the following

Minimize

$$
\iint_{O \times(0, T)} u^{2} d x d t+k \int_{\Omega}\left|y(T)-z_{0}\right|^{2} d x, \quad k>0,
$$

among all couples $(u, y)$ subject to (4.1), (4.2) and (4.3).

Still another possibility is to consider

$$
\inf _{u \in L^{2}(O \times(0, T))} \int_{\Omega}\left|y(T)-z_{0}\right|^{2} d x,
$$

among all couples $(u, y)$ subject to (4.1), (4.2), (4.3). 
Conjecture 1. 1. We conjecture that for Navier-Stokes equations the inf in (4.7) equals 0, i.e. that the attainable set (the set spanned in

$$
H=\left\{\varphi \mid \varphi \in\left(L^{2}(\Omega)\right)^{n}, \operatorname{div} \varphi=0, \varphi \nu=0 \text { on } \Gamma\right\}
$$

by all possible states $(y(T))$ is dense in $H$.

2. It seems possible that the inf in (4.6) decreases as the viscosity $u$ decreases in Navier-Stokes equations.

3. A vague and fuzzy conjecture is that the more "chaotic" is a system, the closest it is from exact controllability.

\section{Instantaneous Sentinel}

Let $y(x, t, \lambda, \tau)=y(\lambda, \tau)$ with $\tau=\left(\tau_{1}, \tau_{2}, \ldots, \tau_{N}\right)$, be the unique solution of the problem

$$
\left\{\begin{aligned}
\frac{\partial y}{\partial t}+(y \nabla) y-\Delta y+f(y) & =-\nabla \Pi+\xi+\lambda \widehat{\xi} & & \text { in } Q \\
\operatorname{divy} & =0 & & \text { in } Q \\
y(0) & =y_{0}+\sum_{i=1}^{N} \tau_{i} \widehat{y}_{0}^{i} & & \text { in } \Omega \\
y & =0 & & \text { on } \Sigma .
\end{aligned}\right.
$$

The function $\xi$ is known. But, the terms : $\lambda \widehat{\xi}$ (so-called pollution term) is unknown, $\widehat{\xi}$ is renormalized and represent the size of pollution.

We denote by

$$
y(x, T ; \lambda, \tau)=y_{o b s}, \forall x \in O,
$$

an observation which is a measure of the concentration of the pollution taken at the fixed time $T$ and on a non empty open subset $O \subset \Omega$ called observatory.

Let $h$ be some function in $L^{2}(O)$, for any control function $u \in L^{2}(O)$, we introduce the functional $S(\lambda, \tau)$ as follows

$$
S(\lambda, \tau)=\int_{\Omega}(h+u) \chi_{O} y(x, T ; \lambda, \tau) d x .
$$

Definition 1. Let $S$ be a real function (5.3) depending only on the parameters $\lambda$ and $\tau$. $S$ is said a sentinel defined by $h$ if the following conditions are satisfied

$$
\left.\frac{\partial S}{\partial \tau}(\lambda, \tau)\right|_{\lambda=0, \tau=0}=0
$$


i.e.

$$
\left.\frac{\partial S}{\partial \tau_{i}}(\lambda, \tau)\right|_{\lambda=0, \tau_{i}=0}=0,1 \leq i \leq N
$$

There exists a control $u \in L^{2}(O)$ such that

$$
\|u\|_{L^{2}(O)}=\min _{\alpha \in U}\|\alpha\|
$$

where $U=\left\{\alpha \in L^{2}(O)\right.$, such that $\left.\left.\frac{\partial S}{\partial \tau}(\lambda, \tau)\right|_{\lambda=0, \tau=0}=0\right\}$.

- Condition (5.4) express insensitivity of $S$ with respect to small variations of $\tau$ and assume the existence of the derivate.

- According to (5.5) which consists in an optimal criterion of selection for (5.4).

We consider the function $y_{0}$ and $\Pi_{0}$ which solve the problem (5.1) for $\lambda=0$ and $\tau_{i}=0$

$$
\left\{\begin{aligned}
\frac{\partial y_{0}}{\partial t}+\left(y_{0} \nabla\right) y_{0}-\Delta y_{0}+f\left(y_{0}\right) & =-\nabla \Pi_{0}+\xi & & \text { in } Q \\
\text { divy } & =0 & & \text { in } Q \\
y_{0}(0) & =y_{0} & & \text { in } \Omega \\
y_{0} & =0 & & \text { on } \Sigma
\end{aligned}\right.
$$

We consider the function $y_{\tau_{i}}$ defined by $y_{\tau_{i}}=\frac{\partial y}{\partial \tau_{i}}(0,0)$, which is the unique solution of the problem

$$
\left\{\begin{array}{rlrl}
\frac{\partial y_{\tau_{i}}}{\partial t}+\left(y_{0} \nabla\right) y_{\tau_{i}}+y_{\tau_{i}}\left(\nabla y_{0}\right)-\Delta y_{\tau_{i}}+f^{\prime}\left(y_{0}\right) y_{\tau_{i}} & =-\nabla \Pi_{\tau_{i}} & \text { in } Q \\
d i v y_{\tau_{i}} & =0 & & \text { in } Q \\
y_{\tau_{i}}(0) & =\widehat{y}_{0}^{i} & & \text { in } \Omega \\
y_{\tau_{i}} & =0 & & \text { on } \Sigma
\end{array}\right.
$$

The condition (5.4) holds if and only if

$$
\int_{\Omega}(h+u) \chi_{O} y_{\tau_{i}}(T) d x=0, \quad 1 \leq i \leq N
$$

In order to transform this equation, we introduce the classical adjoint 
state

$$
\left\{\begin{aligned}
-\frac{\partial q}{\partial t}+\left(y_{0} \nabla\right) q+q\left(\nabla y_{0}\right)-\Delta q+f^{\prime}\left(y_{0}\right)^{*} q & =-\nabla \Psi & & \text { in } Q \\
\operatorname{divq} & =0 & & \text { in } Q \\
q(T) & =(h+u) \chi_{O} & & \text { in } \Omega \\
q & =0 & & \text { on } \Sigma .
\end{aligned}\right.
$$

Theorem 1. Let $q=\left(q_{1}, q_{2}\right)$ be the solution to the backward problem (5.9), then the existence of an instantaneous sentinel insensitive to the missing data is equivalent to the null-controllability problem

$$
\int_{\Omega} q(0) \widehat{y}_{0}^{i} d x=0, \quad 1 \leq i \leq N
$$

i.e

$$
q(0) \in G^{\perp},
$$

where $G^{\perp}$ is the orthogonal of $G$ in $L^{2}(\Omega)$.

Proof. Multiplying the first equation in (5.9) by $y_{\tau_{i}}$, and integrating by parts over $\Omega$, we find

$$
\int_{\Omega} q(0) y_{\tau_{i}}(0) d x-\int_{\Omega} q(T) y_{\tau_{i}}(T) d x=0, \quad 1 \leq i \leq N
$$

then

$$
\int_{\Omega} q(0) \widehat{y}_{0}^{i} d x-\int_{\Omega}(h+u) \chi_{O} y_{\tau_{i}}(T) d x=0, \quad 1 \leq i \leq N,
$$

thanks to (5.8), we have

$$
\int_{\Omega} q(0) \widehat{y}_{0}^{i} d x=0, \quad 1 \leq i \leq N
$$

So that

$$
q(0) \perp \widehat{y}_{0}^{i}, \quad 1 \leq i \leq N .
$$




\subsection{Optimal control problem}

In this section we are interested to solve the problem (5.5), so we consider the optimization problem

$$
u \in M \min \|u\|_{L^{2}(O)}^{2},
$$

with $M=\left\{u \in L^{2}(O)\right.$ such hat, we have (5.4) and $\int_{\Omega} q(0) \widehat{y}_{0}^{i} d x=0$, $1 \leq i \leq N$ where $q$ is the solution of $(5.9)\}$.

Lemma 1. The problem (5.11) admits an unique solution.

Proof. The set $M$ is a non empty, colsed and convex set. The mapping

$$
v \rightarrow\|v\|_{L^{2}(O)}^{2},
$$

is continuous, coercive and strictly convex, therefore, the problem (5.11) admits an unique solution denoted by $\widehat{v} \in M$ which satisfies

$$
\|\widehat{v}\|_{L^{2}(O)} \leq\|v\|_{L^{2}(O)}, \forall v \in M
$$

\subsection{Characterization of optimal control}

To characterize the optimal control, let us introduce $q_{0}$ by

$$
\left\{\begin{aligned}
-\frac{\partial q_{0}}{\partial t}+\left(y_{0} \nabla\right) q_{0}+q_{0}\left(\nabla y_{0}\right)-\Delta q_{0}+f^{\prime}\left(y_{0}\right)^{*} q_{0} & =-\nabla \Psi_{1} & & \text { in } Q \\
\operatorname{div}_{0} & =0 & & \text { in } Q \\
q_{0}(T) & =h \chi_{O} & & \text { in } \Omega \\
q_{0} & =0 & & \text { on } \Sigma .
\end{aligned}\right.
$$

and let us define $z=z(u)$ as the solution of

$$
\begin{aligned}
-\frac{\partial z}{\partial t}+\left(y_{0} \nabla\right) z+z\left(\nabla y_{0}\right)-\Delta z+f^{\prime}\left(y_{0}\right)^{*} z & =-\nabla \Psi_{2} & \text { in } Q \\
\operatorname{div} z & =0 & \text { in } Q \\
z(T) & =u \chi_{O} & \text { in } \Omega \\
z & =0 & \text { on } \Sigma .
\end{aligned}
$$

Then

$$
q=q_{0}+z=q_{0}+z(u), \quad \Psi=\Psi_{1}+\Psi_{2},
$$


we want to find $u$ such that

$$
\int_{\Omega} z(0 ; u) \widehat{y}_{0}^{i} d x=-\int_{\Omega} q_{0}(0) \widehat{y}_{0}^{i} d x, \quad 1 \leq i \leq N .
$$

We define $\rho$ as the solution of

$$
\left\{\begin{aligned}
-\frac{\partial \rho}{\partial t}+\left(y_{0} \nabla\right) \rho+\rho\left(\nabla y_{0}\right)-\Delta \rho & =-\nabla \Xi & & \text { in } Q \\
\operatorname{div} \rho & =0 & & \text { in } Q, \\
\rho(0) & =\sum_{i=1}^{N} \alpha_{i} \widehat{y}_{0}^{i} & & \text { in } \Omega, \\
\rho & =0 & & \text { on } \Sigma .
\end{aligned}\right.
$$

where $\alpha_{i}$ is not determined. Let $\xi$ is the solution of the system

$$
\text { (5.\{6) }\left\{\begin{array}{rlrl}
-\frac{\partial \xi}{\partial t}+\left(y_{0} \nabla\right) \xi+\xi\left(\nabla y_{0}\right)-\Delta \xi+f^{\prime}\left(y_{0}\right)^{*} \xi & =-\nabla \Upsilon & \text { in } Q, \\
\operatorname{div\xi } & =0 & & \text { in } Q, \\
\xi(T) & =\rho(T) \chi_{O} & \text { in } \Omega, \\
\xi & =0 & & \text { on } \Sigma .
\end{array}\right.
$$

and we want to determine $\alpha=\left\{\alpha_{1}, \alpha_{2}, \ldots, \alpha_{N}\right\} \in R^{N}$ such that

$$
\int_{\Omega} \xi(0) \widehat{y}_{0}^{i} d x=-\int_{\Omega} q_{0}(0) \widehat{y}_{0}^{i} d x, \quad 1 \leq i \leq N .
$$

We introduce the linear operator $\Lambda$ by

$$
\Lambda \alpha=\left\{\int_{\Omega} \xi(0) \widehat{y}_{0}^{1} d x, \int_{\Omega} \xi(0) \widehat{y}_{0}^{2} d x, \ldots, \int_{\Omega} \xi(0) \widehat{y}_{0}^{N} d x\right\} .
$$

Then

$$
\Lambda \in L\left(R^{N}, R^{N}\right)
$$

and

$$
\Lambda \alpha=\left\{\int_{\Omega} \alpha \widehat{y}_{0}^{1} d x, \int_{\Omega} \alpha \widehat{y}_{0}^{2} d x, \ldots, \int_{\Omega} \alpha \widehat{y}_{0}^{N} d x\right\} .
$$

Theorem 2. According to the unique continuation theorem of Mizohata [9], we have at least one sentinel given by

$$
S(\lambda, \tau)=\int_{\Omega}(h+\rho(T)) \chi_{O} y(x, T ; \lambda, \tau) d x,
$$

where $\rho$ is the solution of (5.15), so

$$
u=\rho(T) \chi_{O},
$$

is the solution of (5.4)-(5.5). 
Proof. We multiply (5.15) by $\widetilde{\rho}$ corresponding to $\widetilde{\alpha}$, and we integrate by parts. We obtain

$$
\langle\Lambda \alpha, \widetilde{\alpha}\rangle=\int_{O} \rho(T) \widetilde{\rho}(T) d x .
$$

Therefore, $\Lambda$ is a symmetric and positive matrix. Let us now set

$$
\|\alpha\|_{F}=\left(\int_{O} \rho(T)^{2} d x\right)^{1 / 2}
$$

and let $y_{i}(x, t)$ the solution of

$$
\left\{\begin{aligned}
\frac{\partial y_{i}}{\partial t}+\left(y_{i} \nabla\right) y_{i}-\Delta y_{i}+f\left(y_{i}\right) & =-\nabla \Pi_{i} & & \text { in } Q \\
d i v y_{i} & =0 & & \text { in } Q, \\
y_{i}(0) & =\widehat{y}_{0}^{i} & & \text { in } \Omega, \\
y_{i} & =0 & & \text { on } \Sigma .
\end{aligned}\right.
$$

We define in this way a norm on the space $F$ of the functions $\alpha$, where the Hilbert space $F$ is the completion of smooth functions for the norm (5.19) ( indeed if $\|\alpha\|_{F}=0$ then $\rho=0$ on $O$ and according to the unique continuation theorem of Mizohata $\rho=0$ on $Q$ so that $\alpha=0$ ). Then if $F^{\prime}$ denotes the dual of $F$, we have

$$
\Lambda: F \longrightarrow F^{\prime} \text { is an isomorphism. }
$$

Therefore, the equation

$$
\Lambda \alpha=-\left\{\int_{\Omega} q(0) \widehat{y}_{0}^{1} d x, \int_{\Omega} q(0) \widehat{y}_{0}^{2} d x, \ldots, \int_{\Omega} q(0) \widehat{y}_{0}^{N} d x\right\},
$$

admits a unique solution if

$$
-\int_{\Omega} q(0) \widehat{y}_{0}^{i} d x \in F^{\prime}, \quad 1 \leq i \leq N .
$$

We set

$$
\beta=\left\{\int_{\Omega} q(0) \widehat{y}_{0}^{1} d x, \int_{\Omega} q(0) \widehat{y}_{0}^{2} d x, \ldots, \int_{\Omega} q(0) \widehat{y}_{0}^{N} d x\right\},
$$

then the solution of (5.20) is given by

$$
\alpha=-\Lambda^{-1} \beta \text {. }
$$

If we multiplying (5.12) by $\rho$, and integrating over $Q$ we obtain

$$
u=\rho(T) \chi_{O},
$$

is the solution of (5.5), (5.10).

Remark 3. The space $F$ is identical to $R^{N}$, and its norm is equivalent to the Euclidian norm. Then, $\Lambda$ is a isomorphism from $R^{N}$ to $R^{N}$. 


\subsubsection{Identification of the pollution term}

Let $S_{o b s}$ be the measured sentinel corresponding to the state of the system on the observatory $O$ at the time $T$

$$
S_{o b s}(\lambda, \tau)=\int_{\Omega}(h+u) \chi_{O} y_{o b s}(x, T ; \lambda, \tau) d x .
$$

Theorem 3. The pollution term is estimated as follows

$$
\int_{0}^{T} \int_{\Omega} q(h) \lambda \widehat{\xi} d x d t=S_{o b s}(\lambda, \tau)-S(0,0),
$$

where $S(0,0)$ is the sentinel corresponding to the state $y(x, T ; 0,0)$.

Proof. We have

$\left(5.85 h_{s}(\lambda, \tau)=S(0,0)+\left.\lambda \frac{\partial S}{\partial \lambda}\left(\lambda, \tau_{i}\right)\right|_{\lambda=0, \tau_{i}=0}+\circ\left(\lambda, \tau_{i}\right)\right.$, for $\lambda, \tau$ small,

and

$$
\frac{\partial S}{\partial \lambda}(\lambda, \tau)=\int_{O}(h+u) y_{\lambda} d x
$$

where $y_{\lambda}$ defined by $y_{\lambda}=\frac{\partial y}{\partial \lambda}(0,0)$ ( which depends only on $\widehat{\xi}$ and the other known data) is the unique solution of

$$
\left\{\begin{array}{rlrl}
\frac{\partial y_{\lambda}}{\partial t}+\left(y_{0} \nabla\right) y_{\lambda}+y_{\lambda}\left(\nabla y_{0}\right)-\Delta y_{\lambda}+f^{\prime}\left(y_{0}\right) y_{\lambda} & =-\nabla \Pi_{\lambda}+\widehat{\xi} & \text { in } Q \\
\operatorname{div} y_{\lambda} & =0 & & \text { in } Q \\
y_{\lambda}(0) & =0 & & \text { in } \Omega, \\
y_{\lambda} & =0 & & \text { on } \Sigma
\end{array}\right.
$$

and

$$
\left.\lambda \frac{\partial S}{\partial \lambda}(\lambda, \tau)\right|_{\lambda=0, \tau=0}=S_{o b s}(\lambda, \tau)-S(0,0) .
$$

We designate by $q(h)$ the unique solution of (5.9) depending on $h$.

Multiply (5.9) by $y$ and integrate by part, we obtain

$$
\int_{\Omega}(h+u) \chi_{O} y_{\lambda} d x=\int_{0}^{T} \int_{\Omega} q(h) \lambda \widehat{\xi} d x d t .
$$

Therefore, the pollution term can be characterized

$$
\int_{0}^{T} \int_{\Omega} q(h) \lambda \widehat{\xi} d x d t=S_{\text {obs }}(\lambda, \tau)-S(0,0) .
$$




\subsection{Conclusion}

In this paper we are working to get instantaneous information at fixed instant $T$ on pollution term in Navier-Stokes system in which the initial condition is incomplete. The best method which can solve this problem is the sentinel method; it allows estimating the pollution term at which we look for information independently of the missing term that we do not want to identify. So, we prove the existence of such instantaneous sentinel by solving a problem of controllability with constraint on the control. From this we conclude that there is a harmony between the theory of turbulence and sentinel with the theory of control.

\section{Acknowledgements}

The authors thank the referee for his helpful comments and suggestions.

This work was supported by the Directorate-General for Scientific Research and Technological Development (DGRSDT).

\section{References}

[1] A. Berhail and I. Rezzoug, "Identification of the source term in Navier-Stokes system with incomplete data", AIMS mathematics, vol. 4, no. 3, pp. 516-526, 2019. https:// doi.org/ 10.3934/ math.2019.3.516

[2] A. El-Jai, Eléments de contrôlabilité. Perpignan: Presses universitaires de Perpignan, 2006.

[3] A. Mellet and A. Vasseur, "Existence and uniqueness of global strong solutions for one-dimensional compressible navier-stokes equations", SIAM journal on mathematical analysis, vol. 39, no. 4, pp. 1344-1365, 2008.

[4] C. L. Fefferman, "Existence \& smoothness of the Navier-Stokes equation," Clay Mathematics Institute, 16-Nov-2021. [Online]. Available: https:/ / bit.ly/ 3nLLQoo

[5] G. G. Chavent, "Generalized sentinels defined via least squares", Applied mathematics \& optimization, vol. 31, no. 2, pp. 189-218, 1995. https:/ / doi.org/ 10.1007/ BF01182788

[6] I. Rezzoug, T. E. Oussaeif, and A. Benbrahim, "Solvability of a solution and controllability for nonlinear fractional differential equations", Bulletin of the Institute of mathematics, vol. 15, no. 3, pp. 237-249, 2020. https:/ / doi.org/ 10.21915/ BIMAS.2020303 
[7] I. Rezzoug and O. Taki-Eddine, "Approximate controllability", WSEAS transactions on systems, vol. 19, pp. 14-19, 2020. https:/ / doi.org/ g63f

[8] I. Rezzoug and A. Ayadi, "Weakly sentinel involving a navier-stokes problem and detecting pollution", General letters in mathematics, vol. 5, no. 2, pp. 93-104, 2018. https:/ / doi.org/ 10.31559/ glm2018.5.2.4

[9] I. Rezzoug, "Etude théorique et numérique des problèmes d'identification des systèmes gouvernés par des équations aux dérivées partielles", Thèse de doctorat, Université de Oum El Bouaghi, Algérie, 2014. 2014. https:/ / bit.ly/ 3FMeeN8

[10] I. Rezzoug, "Identification d'une partie de la fronti ere inconnue d'une membrane", Thèse de magister, Université de Oum El Bouaghi, Algérie, 2009. https:// bit.ly/ 32q3pSB

[11] E. Miot, "Solutions lagrangiennes ou singulières des équations de Vlasov-Poisson et d'Euler: existence, unicité, interactions et collisions", Mémoire d'habilitation à diriger des recherches, Université Grenoble Alpes, 2019. https:/ / bit.ly/ 3COTouO

[12] E. Zuazua, "Exact controllability for semilinear wave equations in one space dimension", Annales de l'Institut Henri Poincaré. Analyse non linéaire, vol. 10, no. 1. pp. 109-129, 1993. https:/ / bit.ly/ 3HRe2Ot

[13] J.-P. Kernevez, The sentinel method and its application to environmen- tal pollution problems. Boca Raton, FL: CRC Press, 1997.

[14] J. L. Lions, "Insensibilization of functionals connected with distributed systems”, Conference Rambouillet, 1989.

[15] J. L. Lions, Sentinelles pour les systèmes distribués à Données incomplètes. Paris: Masson, 1992.

[16] K. Ines and A. Ayadi, and I. Rezzoug, "Weak controllability and the new choice of actuators", Global journal of pure and applied mathematics, vol. 14, no. 2, pp. 325-330, 2018 
[17] L. Teldja, I. Rezzoug, and A. Berhail, "Approximate controllability of the Stokes system", Global journal of pure and applied mathematics, vol. 14, no. 6, pp. 775-785, 2018.

[18] D. C. Leslie, "Turbulence in fluids. by M. L ESIEUR. Martinus Nijhoff, 1987. 286 pp. DFL155.00 or £4.75.", Journal of fluid mechanics, vol. 194, pp. 598-599, 1988. https:/ / doi.org/ 10.1017/ S0022112088213131.

[19] O. A. Ladyzhenskaya, "Sixth problem of the millennium: Navier-Stokes equations, existence and smoothness", Uspekhi Matematicheskikh Nauk, vol. 58, no. 2, pp. 45-78, 2003.. https:/ / doi.org/ 10.4213/rm610.

\author{
Laib Teldja \\ Department of Mathematics, \\ Laboratory of Dynamical Systems and Control, \\ University of Larbi Ben M'hidi Oum El Bouaghi, \\ P. O. Box 358, OEB, \\ Algeria \\ e-mail: teldmaths@hotmail.com

\section{Rezzoug Imad} \\ Department of Mathematics, \\ Laboratory of Dynamical Systems and Control, \\ University of Larbi Ben M'hidi Oum El Bouaghi, \\ P. O. Box 358, OEB, \\ Algeria \\ e-mail: imad.rezzoug@univ-oeb.dz \\ Corresponding Author \\ and

\section{Oussaeif Taki-Eddine} \\ Department of Mathematics, \\ Laboratory of Dynamical Systems and Control, \\ University of Larbi Ben M'hidi Oum El Bouaghi, \\ P. O. Box 358, OEB, \\ Algeria \\ e-mail: taki maths@live.fr
}

